\title{
O USO DO WORLD CAFÉ COMO MÉTODO DE PESQUISA JUNTO ÀS EQUIPES DE SAÚDE
}

\section{The use of world café as a research method among health teams}

\author{
Marcelo Pedra Martins Machado \\ Escola Fiocruz de Governo - Fundação Oswaldo Cruz - Brasília
}

Maria Fabiana Damásio Passos

Escola Fiocruz de Governo - Fundação Oswaldo Cruz - Brasília

\section{RESUMO}

Objetivo: Apresentar as características do World Café, identificando os principais aspectos que o torna uma estratégia de coleta de dados qualitativa que contribui para a interação e estimula a participação dos sujeitos de pesquisa. Métodos: Para tanto, foi utilizado como ponto de partida a utilização da referida técnica junto às equipes de saúde que atuam com pessoas em situação de rua no Distrito Federal - Brasil. Resultados e Conclusão: Verificou-se que o World Café foi de grande relevância para a discussão de temas que são considerados balizadores para as práticas das equipes.

Descritores: World Café; Equipes de Saúde; Políticas Públicas; Ação Interativa.

\section{ABSTRACT}

Objective: The present work aims to present the characteristics of the World Café, identifying the main aspects that make it a qualitative data collection strategy that contributes to the interaction and stimulates the participation of the research subjects. Methods: Therefore, the use of this technique was used as a starting point for the health teams that work with street people in the Federal District - Brazil. Results and Conclusion: It was verified that the World Café was of great relevance for the discussion of topics that are considered as guides for the practices of the teams.

Descriptors: World Café; Public Health; Health Team; Interaction.

\section{INTRODUÇÃO}

O world café é uma técnica que vem sendo utilizada em vários países com o objetivo de potencializar diálogos e viabilizar a construção coletiva de proposições em torno de temas relevantes nos diversos campos de conhecimento. Visa ao estabelecimento de um espaço dialógico que, por meio de perguntas, estimula a participação das pessoas na emissão de opiniões e construção coletiva de entendimento sobre um objeto que é apresentado como cerne para a discussão (Brown \& Isaacs, 2008).

Para realizar o world café, é importante atentar-se para os seguintes princípios: definição do contexto, criação de um ambiente acolhedor, estabelecimento das questões importantes a serem exploradas, encorajamento para que todos possam dar sua contribuição, conexão das diversas perspectivas colocadas pelos participantes, escuta coletiva das ideias e dos insigths e compartilhamento das ideias (The world Café Community, 2015).

De acordo com o documento produzido pelo The World Café Community (2015), para viabilizar a realização do world café, é necessário estruturar grupos de aproximadamente quatro pessoas para a discussão de temas que sejam elencados como importantes (por via da literatura ou dos próprios participantes) para a compreensão de um determinado objeto de estudo ou de trabalho. Uma vez separados em subgrupos, são destinados aos participantes 
em torno de vinte a trinta minutos para realizarem exposição das suas ideias a respeito dos temas disparadores das rodadas progressivas de diálogo, disponíveis em cada mesa de discussão.

Para cada mesa, é escolhido um anfitrião que é indicado como responsável pelo registro das questões mais relevantes discutidas pelo grupo durante o tempo determinado para tal. Essa escolha pode ocorrer no início ou no final da discussão, a depender da dinâmica definida pelo condutor do processo.

Um dos diferenciais do world café consiste em garantir a agilidade e fluidez entre os grupos, de modo a permitir que os participantes transitem por diversos arranjos grupais e não permaneçam com as mesmas pessoas até o final da atividade. O mais relevante é que haja a circulação das ideias por parte de quem segue para os demais grupos (nos diferentes temas) e a preservação do que já fora discutido para que seja utilizado como insumo entre os demais que aterrissam naquele determinado ponto a ser discutido. Assim, o anfitrião de cada mesa de debate tem um papel fundamental de acolher os participantes que chegam para iniciar o debate e de realizar a conexão entre as ideias expostas a respeito de um determinado tema.

Segundo Brown \& Isaacs (2008), vinculados ao The World Café Community, outro ponto de grande relevância desta técnica é permitir a conexão dos diversos temas que são debatidos em torno de um único objeto. Essa interlocução entre os temas é viabilizada por conta da circulação dos participantes, que pode emitir opiniões sobre os temas debatidos pelos demais grupos.

Para que as discussões no world café possam fluir de modo produtivo, é importante que os responsáveis pela sua condução criem um ambiente que seja convidativo para a reflexão e marcado pela segurança e confiança para expor suas visões e posicionamentos. Segundo os autores, o cerne do trabalho com o world café está na participação ativa dos membros dos grupos e tem como consequência a construção de uma inteligência coletiva a respeito do tema elencado para o debate. Portanto, ao longo da sua realização, estimular a fala e a exposição das ideias e dos contrapontos entre os participantes é uma tarefa importante e que merecerá a atenção para quem está conduzindo o processo.

O world café vem sendo largamente utilizado por pesquisadores por se tratar de uma técnica propositiva, que permite a construção de diferentes horizontes do tema que se define para o trabalho. Como exemplo, pode-se citar a experiência relatada por Mandeli, Roland, Souza, Germani, Gouveia \& Dias (2013), em que o world café foi utilizado para identificar as características do trabalho do catador de material reciclável e suas necessidades de saúde. Os resultados do uso do world café, segundo os autores, propiciaram a construção de projetos de pesquisa para o reconhecimento de modo mais efetivo da realidade dos catadores, buscando garantir a sua valorização profissional.

O presente trabalho tem como objetivo relatar a experiência de uso da técnica do world café como estratégia de coleta de dados em uma pesquisa qualitativa no campo da saúde pública, de modo a destacar as vantagens e desvantagens da sua utilização. No caso em questão, a referida técnica foi utilizada em uma pesquisa que tinha por objetivo analisar o processo de trabalho de equipes de consultório na rua (eCR), realizada por um aluno do Mestrado em Políticas Públicas em Saúde da Fundação Oswaldo Cruz - Brasília, um dos autores deste artigo.

Assim, buscou-se, por meio do world café, estimular os trabalhadores a verbalizarem sobre os sentimentos, motivações, crenças, valores, relações sociais e significados (Serapioni, 2005) construídos em torno da realização das suas atividades junto à PSR. A referida técnica foi utilizada ainda como um caminho de possibilidades para a colaboração e construção de perspectivas sobre a realidade dos trabalhadores em questão, indicando coletivamente meios para a sua transformação.

Nessa direção, o manuscrito apresenta uma breve descrição das principais características do world café e em seguida faz um apanhado dos resultados mais relevantes proporcionados com a sua utilização junto aos trabalhadores das eCR.

\section{MÉTODOS}

A escolha pelo world café se deu pela possibilidade de aprofundamento das questões simbólicas, da subjetividade e da aproximação entre pesquisador e participante da pesquisa (Minayo \& Sanches, 1993), dado que a técnica se dá estimulando o diálogo e o compartilhamento das experiências para o entendimento do escopo das atividades desenvolvidas pelos trabalhadores das eCR em um contexto marcado pela adversidade advinda da realidade da População em Situação de Rua - PSR. Além disso, o uso da abordagem qualitativa buscou permitir o debate entre os trabalhadores das eCR sobre o processo de implementação das políticas públicas recentes que propõem diretrizes para as práticas destas equipes, assim como o entendimento sobre o processo de trabalho deles a partir da interlocução entre as referidas políticas e a realidade enfrentada. 
Participaram do world café 14 profissionais de saúde que atuam em equipes de consultório na rua no Distrito Federal. Tais profissionais foram organizados no território em equipes conforme deliberação das portarias 122/2012 e 123/2012 do Ministério da Saúde do Brasil, que definem como seus objetivos oportunizam acesso e atenção integral aos diferentes problemas e necessidades de saúde da população em situação de rua, assim como definem também os profissionais que podem compor estas equipes. Além disso, definem as modalidades de equipe, em função da população que demanda atendimento por município.

A referida atividade é parte de uma dissertação de mestrado em Políticas Públicas em Saúde, constituindose neste contexto como uma das três estratégias de coleta de dados (junto com entrevistas semiestruturadas e questionários) utilizadas para o conjunto de análises apresentadas pela dissertação. Portanto, a condução do world café foi realizada pelo mestrando e sua orientadora, ambos atuantes no campo da saúde pública.

Os trabalhadores foram distribuídos em três mesas reservadas para a discussão de três diferentes temas vulnerabilidade, resolutividade e indicadores - que serviram como referência para a compreensão do processo de trabalho das equipes de consultório na rua, no Distrito Federal, foco da pesquisa. Para viabilizar a discussão, os trabalhadores foram acomodados em dois grupos de cinco e um grupo com quatro participantes.

Para iniciar a atividade, foi estabelecida como aquecimento a realização de uma rodada sobre os objetivos e funções da eCR. Os três grupos discutiram o mesmo tema com o objetivo de entender a metodologia.

As rodadas progressivas de diálogo aconteceram com duração de 20 minutos cada com perguntas ou questões que referentes ao eixo trabalhado, por meio dos quais os participantes de cada grupo foram estimulados a expor e registrar suas opiniões, além dos principais consensos e dissensos construídos por meio do debate.

Foram reconhecidas como rodadas progressivas porque os registros foram utilizados pelos grupos subsequentes como forma de aquecimento e apresentados pelo anfitrião escolhido pela mesa, que ficou responsável por transmitir as ideias anteriores e fazer as interlocuções necessárias para a construção das ideias coletivas.

Ao final, foi realizada uma plenária para apresentação dos produtos discutidos em cada um dos grupos e para que os trabalhadores pudessem reconhecer seus posicionamentos e para a definição de possíveis encaminhamentos.

Todos os participantes assinaram o Termo de Consentimento Livre e Esclarecido (TCLE), que informou sobre os objetivos da pesquisa e a garantia de sigilo no processo de participação. Foi solicitada ainda autorização para gravação e para uso de imagem.

As três categorias trabalhadas, foram escolhidas com base na revisão de literatura realizada para o referido estudo, no surgimento dos referidos temas em reuniões anteriores ao world café com os participantes e na apresentação da pesquisa para os responsáveis técnicos e gestores. Além disso, as referidas categorias foram reforçadas nas entrevistas realizadas com os gestores das eCR, no Distrito Federal.

As questões disparadoras para o world café no que se refere ao eixo vulnerabilidade foram relacionadas ao entendimento do grupo sobre o termo (inserido no processo de trabalho) e sobre as principais situações de vulnerabilidade encontradas pelos trabalhadores e como lidam com essa situação.

No eixo resolutividade, as questões também foram elaboradas para analisar o entendimento que os trabalhadores tinham sobre o termo (referido ao processo de trabalho), na interferência da rede local sobre a resolutividade da equipe e quais os efeitos da eCR. Para as discussões sobre o eixo de indicadores, as questões disparadoras foram sobre a existência de parâmetros de avaliação do trabalho da eCR, quais os melhores indicadores para avaliar a eCR (processo e resultado) e sobre como os indicadores podem ser incorporados no cotidiano da equipe.

Em síntese, para cada categoria de análise, foi indicado um tema norteador. Assim, os três temas foram distribuídos pelas mesas da seguinte forma: Mesa 1 - Vulnerabilidade: "concepção e formas de lidar vulnerabilidade"; Mesa 2 - Resolutividade: "concepção de resolutividade e interlocução com a rede"; Mesa 3 - Indicadores: "indicadores de avaliação do processo de trabalho".

Para a realização do world café, foram estabelecidas três categorias de análise: vulnerabilidade, resolutividade e indicadores. A noção de vulnerabilidade indica suscetibilidades das pessoas a problemas e/ou danos de saúde, identificando indivíduos, grupos e comunidades que estão expostos a condições de maiores níveis de risco nos planos sociais, políticos e econômicos - condições essas que afetam a vida individual, familiar e comunitária (Bertolozzi, Nichiata, Takahashi, Ciosak, Hino, Val, Guanillo e Pereira, 2009).

Deste modo, o conhecimento sobre a vulnerabilidade aos agravos de saúde ganha relevância nos efeitos e implicações que produz na saúde das pessoas que estão vulneráveis e, por consequência, na identificação das suas demandas e necessidades de saúde, contribuindo como elemento de análise para que sejam construídos e assegurados meios de proteção. Há também leituras que problematizam o conceito de vulnerabilidade. De um 
modo geral há quase um consenso entre os autores citados anteriormente que a vulnerabilidade está ligada à ideia de "defeito", algo que deve ser eliminado.

Na sociedade capitalista contemporânea, a vulnerabilidade é sinônimo de fracasso. Deste modo, o vulnerável é tomado e produzido como fracassado. Nessa perspectiva, diante do vulnerável inicia-se uma operação, por diferentes segmentos da sociedade (incluindo os serviços públicos), pela eliminação da diferença que o vulnerável introduz, seja colocando-o em "seu lugar" de direito, remodelando-o, readaptando-o ou mesmo restituindo seu lugar na sociedade. O conceito de vulnerabilidade pode se tornar mais interessante e produtivo se pensado não como atributo de um objeto, algo separado de nós, mas como uma vulnerabilidade nossa, de todos.

Segundo Monteiro (2011), uma situação de vulnerabilidade abrange múltiplos condicionantes, não se restringindo a uma pessoa ou grupo, mas sim a determinadas condições e circunstâncias que poderiam ser revertidas.

Já a noção de resolutividade tem relação direta com o ato de dar solução a alguma coisa. Segundo Turrini, Lebrão \& Cesar (2008), a resolutividade é considerada como a resposta satisfatória que um serviço de saúde oferece ao usuário do SUS, quando este procura atendimento para alguma necessidade de saúde. Essa resposta não abrange somente a cura de doenças, mas inclui o alívio ou a minimização do sofrimento, assim como a prevenção de outros agravos ou doenças, a promoção e a manutenção da saúde dos indivíduos e coletivos.

A ação resolutiva, segundo Merhy (1994) não se reduz a uma conduta. O autor afirma que resolutividade é quando colocamos à disposição do usuário um conjunto de tecnologias disponíveis com o objetivo de promover o diagnóstico e o tratamento adequado a cada caso, contemplando as dimensões individuais e coletivas dos problemas de saúde.

Deste modo, a intervenção efetiva em saúde necessita de uma abordagem integral dos problemas. Este autor nos alerta para a associação entre as ações coletivas e as individuais, pois na atualidade os problemas de saúde transcendem, na sua maioria, a questão individual. Outro ponto importante para abordar a questão da resolutividade é o trabalho em equipe. Para o profissional, a possibilidade de uma prática colaborativa, com troca de saberes e experiências clínicas entre as diversas categorias profissionais e experiências, possibilita maior resolutividade para os problemas apresentados pelos sujeitos e também pela comunidade (Travassos, 2004).

Pensar a questão da resolutividade na $\mathrm{AB}$ exige uma reflexão sobre capacidade do sistema de saúde como um todo, com seus limites de complexidade e capacidade tecnológica de resolver os problemas de saúde que levam o usuário a procurar os seus serviços, em cada nível de atenção. Assim como há de se refletir sobre os problemas que causam impacto coletivo à saúde da população, a partir do ideário/lógica de que serviços têm seu funcionamento e organização a partir de um território específico (Chioro e Scaff, 1999).

De um modo geral, não há consenso no Brasil sobre o que se espera em termos da resolutividade dos serviços da AB. Além disso, a formação de recursos humanos segue sendo um desafio para o SUS e para a AB. A medicina de família e comunidade só foi reconhecida como especialidade médica em 2002. Há também a baixa credibilidade e reconhecimento dos profissionais de $A B$ pela rede de saúde com relação à resolutividade, elemento que se torna um obstáculo para coordenação dos cuidados a partir da AB (Almeida, Fausto e Giovanella, 2011).

Podemos considerar que os indicadores são medidas-síntese que apresentam informações relevantes sobre determinadas propriedades do estado de saúde, além de informar sobre como caminha o desempenho do sistema de saúde (OPAS, 2008).

Outra forma de definir os indicadores de saúde são como informações construídas com periodicidade definida, realizada com base nos dados coletados pelos sistemas de informação. Outra forma de conceituar os indicadores de saúde é como parâmetros de utilização internacional que objetivam avaliar, do ponto de vista sanitário, a saúde dos coletivos humanos, assim como subsidiar o planejamento em saúde, possibilitando o acompanhamento das alterações e tendências históricas do padrão sanitário de diferentes coletividades (Rouquayrol, 1993).

A qualidade de um indicador tem relação direta com as características dos elementos utilizados em sua construção (tamanho da população em risco, frequência de casos) e também do quão precisos são os sistemas de informação utilizados para esta construção (coleta, registro, transmissão dos dados). A validade de um indicador é definida por sua capacidade de aferir/medir o que se pretende e confiabilidade (reproduzir os mesmos resultados quando aplicado em condições similares). Em situação ideal o indicador é claro e compreensivo para todos (trabalhadores de saúde, gestores e controle social) (OPAS, 2008).

A confiança dos usuários na informação produzida é construída a partir do monitoramento da qualidade dos indicadores, além da revisão periódica da consistência da série histórica de dados e da disseminação da informação de forma regular (Rouquaryol, 1993). 


\section{RESULTADOS}

Os 14 participantes do world café tinham idades que variavam entre 28 e 44 anos, apresentando média de 34,4 anos (DP=6,24). Conforme dados apresentados na Tabela I, os participantes apresentaram formações diversas e consonantes com as exigências estabelecidas para a estruturação de equipes de consultório na rua.

O tempo médio de formação dos participantes é de 8,9 anos $(\mathrm{DP}=4,9)$ e o tempo médio de integração às eCR é de 2,45 anos $(\mathrm{DP}=1,7)$. $\mathrm{O}$ trabalhador que e apresentou o maior tempo está na eCR há 5 anos, corroborando com o processo de instalação das equipes após a publicação das suas portarias.

Tabela I - Formação dos participantes.

\begin{tabular}{lc}
\hline Formação & $\mathbf{n}$ \\
\hline Psicologia & 2 \\
Pedagogia & 1 \\
Enfermagem & 3 \\
Serviço Social & 2 \\
Medicina & 3 \\
Técnico de Enfermagem & 3 \\
\hline
\end{tabular}

As três rodadas de discussão sobre os temas propostos compuseram a aplicação da técnica do world café, estimulando a troca entre os participantes, a partir das suas vivências em suas realidades de trabalho e permitindo que eles fizessem uma síntese dos principais aspectos discutidos.

Assim, o anfitrião de cada mesa ficou com a atribuição de elencar os pontos mais relevantes para cada tema. Para a discussão sobre os objetivos e funções das equipes, os principais pontos elencados como relevantes foram o acesso ao atendimento, a redução de danos, o estabelecimento de vínculo com a população, o conhecimento da realidade da rua e o desenvolvimento de ações de promoção da saúde.

Já para a discussão sobre vulnerabilidade, os trabalhadores destacaram a falta de garantia de acesso às políticas públicas para a PSR, a ausência de condições econômicas para viabilizar a mobilidade para o atendimento e a vulnerabilidade da própria equipe para continuar o atendimento em condições adversas. Quanto à resolutividade, os trabalhadores destacaram o matriciamento e o trabalho em rede como estratégias de garantia de transferência de conhecimento, de troca de experiências e de ampliação do acesso.

No último item discutido, os indicadores, a síntese feita pelos profissionais destaca a necessidade de mensurar os seguintes aspectos: produtividade, adesão, acessibilidade, melhoria da condição de saúde, inserção na rede e autonomia dos usuários. Vale ressaltar que estes resultados são preliminares e compõem um conjunto maior de análises não apresentados neste trabalho que são fruto da interação dos achados apresentados neste trabalho com outras duas coletas (entrevistas e questionários).

\section{Síntese dos pontos elencados no processo de café mundial}

O produto final do Café Mundial foi um conjunto de elementos escolhidos e sistematizados dentre os vários pontos e temas que emergiram e foram sendo desenvolvidos ao longo do processo de trocas e discussões proposto pelo método.

O debate sobre o processo de trabalho das equipes, construído a partir dos três eixos de discussão (vulnerabilidade, resolutividade e indicadores) proporcionou um resultado apresentado nos Quadros 1, 2 e 3. Além destes, o tema proposto como aquecimento também foi sistematizado pelos trabalhadores, portanto, avalia-se que é pertinente que ele faça parte deste estudo e componha o conjunto de comentários e análises (Quadro 4).

Todos os 4 (quatro) quadros representam a transcrições da síntese construída de forma coletiva pelos trabalhadores dos Consultórios na Rua do Distrito Federal durante as rodadas do Café Mundial e validadas por todos no momento da plenária final. Os itens aparecem na ordem estabelecida pelos grupos de trabalho. Não houve, por parte dos pesquisadores, mudança nos termos usados pelos trabalhadores.

Retornando aos achados desta fase da pesquisa, o Quadro I mostra a produção do grupo de trabalhadores sobre o tema da vulnerabilidade. 


\section{Quadro I - Síntese sobre a vulnerabilidade}

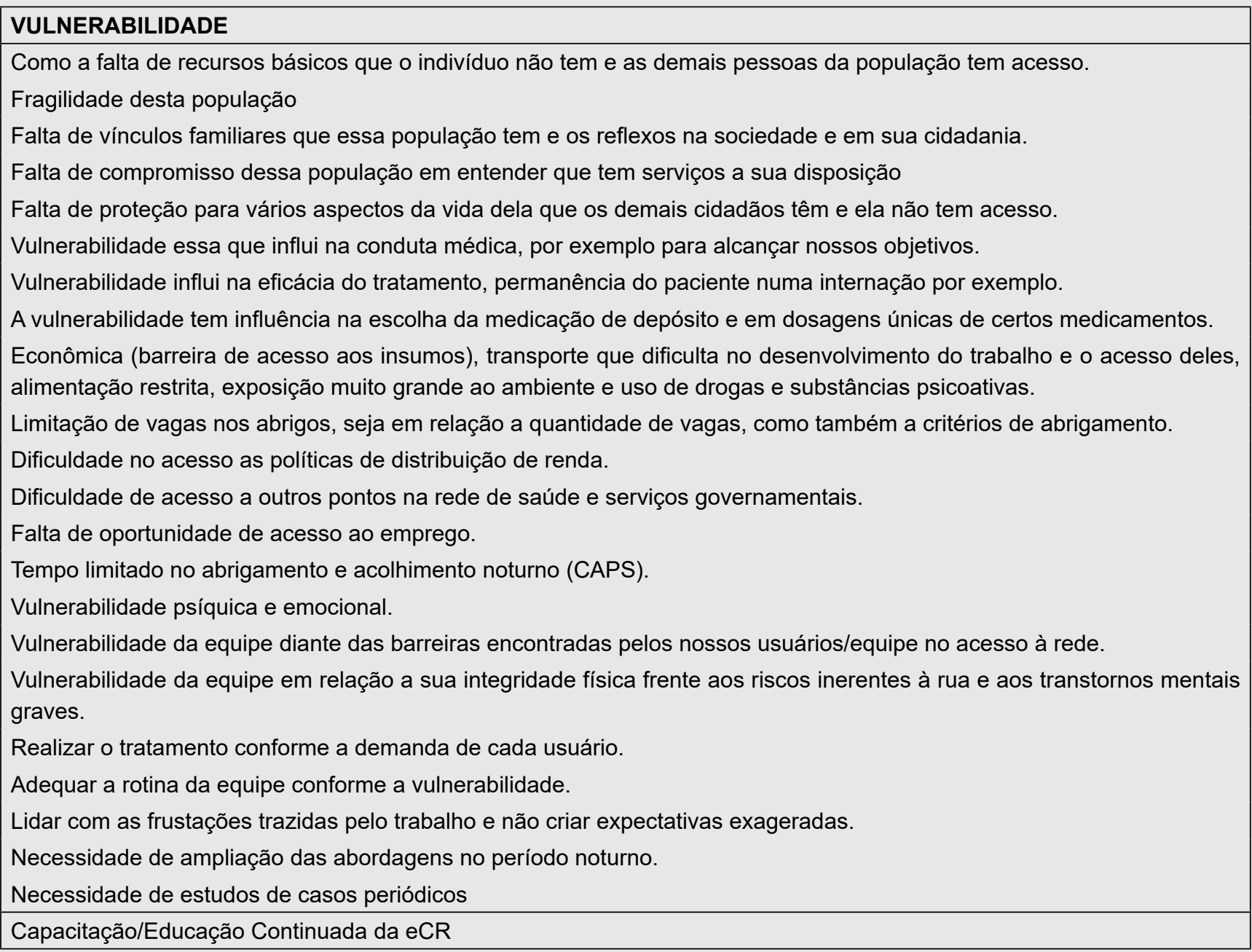

Fonte: Machado, 2017.

Na síntese apresentada no Quadro I, a vulnerabilidade é concebida de inúmeras formas e articulada com diferentes temas pelos trabalhadores das eCR. A falta de acesso a recursos básicos de subsistência, de proteção, a falta de vínculos familiares, a falta de oportunidade de emprego, a fragilidade psíquica e emocional, as restrições econômicas e alimentares e a grande exposição a ambientes com a presença de substancias psicoativas foram algumas das formas apontadas como elementos para compor o entendimento sobre o conceito de vulnerabilidade no olhar dos trabalhadores das eCR/DF. Há nestas leituras uma validação da proposta de ampliação do conceito de vulnerabilidade para além do âmbito individual (Macareta, 2015), colocando a dimensão coletiva deste processo em destaque.

A vulnerabilidade foi articulada com a clínica produzida por estas equipes, assim como com o processo de trabalho das eCR na síntese final sobre o tema quando os trabalhadores associaram a influência da vulnerabilidade na conduta médica, na eficácia do tratamento, na permanência do paciente numa internação, na escolha das medicações ou mesmo das dosagens das mesmas. Além disso, emergiu também a necessidade de um acolhimento diferenciado, levando em consideração os diferentes graus de vulnerabilidade e a necessidade de ampliar as abordagens no período noturno.

A relação dos Consultórios na Rua com a rede local também aparece na síntese como um ponto crítico na construção do pensamento sobre o tema da vulnerabilidade, citando nesse aspecto a limitação de vagas nos abrigos, a imprecisão nos critérios para inclusão no processo de abrigamento institucional e na limitação do tempo de permanência nestes serviços, a dificuldade do acesso da PSR pela eCR às políticas de distribuição de renda, a dificuldade de acesso da PSR atendida pela eCR a outros pontos na rede de saúde e serviços governamentais, a limitação de tempo no acolhimento noturno nos CAPS e a necessidade, por conta do conjunto de dificuldades descritas na relação com a rede, de sensibilizar a rede local para o problema como um todo. 
A vulnerabilidade dos próprios trabalhadores e das equipes foi outro ponto que acumulou elementos neste debate, em que foram citadas as poucas garantias da integridade física da equipe na relação com os riscos inerentes à rua e os transtornos mentais graves, assim como a vulnerabilidade da equipe para superar as barreiras de acesso encontradas pelos usuários/equipe na relação com a rede.

O produto das discussões e abordagens sobre o conceito de resolutividade, realizadas pelos trabalhadores das eCR/DF são apresentados no Quadro II.

Quadro II - Síntese sobre a resolutividade.

\section{RESOLUTIVIDADE}

Ações conclusivas conforme a demanda e aceitação da PSR

Possibilitar a construção de relação com o usuário que favoreça o acompanhamento e a autonomia - vínculo.

Quantas pessoas atendeu sem precisar encaminhar

Matriciamento aumenta a resolutividade pela transferência de conhecimento de outros profissionais.

Encaminhamento para especialistas com referência e contra referência.

Relação com a rede interfere de maneira direta visto que oferta de assistência não supre a demanda, seja por falta de estrutura, inclusive por falta de esclarecimento por parte dos servidores.

Importância da participação da comunidade (rede ampliada).

Aproximação da PSR com a rede ampliada e vice-versa.

Garantia do exercício com equidade (possibilita o exercício de direitos).

Fonte: Machado, 2017.

O Quadro II nos apresenta como o tema da resolutividade ganhou contorno através da síntese final proposta. Os trabalhadores das eCR consensuaram alguns entendimentos sobre o termo resolutividade que são simples e diretos, afirmando-o como ações conclusivas da eCR junto à PSR ou mesmo uma relação entre pessoas atendidas e encaminhamentos, uma leitura próxima a literatura que considera resolutividade como a resposta satisfatória que um serviço de saúde oferece quando um usuário do SUS procura por atendimento (D’Aguiar, 2001).

Além de descrições mais assertivas, outros pontos foram referidos na relação com a resolutividade, entre eles a necessidade da eCR relacionar construção de vínculo, o acompanhamento, a produção de autonomia do usuário e resolutividade, a interferência da relação da eCR com a rede e a construção de ofertas mais resolutivas, a institucionalização das formas de referenciar e contrarreferenciar a PSR junto à rede e sobretudo uma aposta no matriciamento como estratégia de aumento da resolutividade da equipe e da qualificação dos profissionais. Estas leituras são mais próximas à perspectiva colocada por Merhy (1994), que coloca a ação resolutiva não reduzida a uma conduta. Deste modo, para este autor, a efetividade da ação estará sempre pautada pela lógica da abordagem integral dos problemas. Por outro lado, fica apontada também pela síntese dos trabalhadores a necessidade de produção de intersetorialidade como determinante para a ampliação da resolutividade (D’Aguiar, 2001), assim como a existência de uma rede hierarquizada, organizada e articulada no compartilhamento do cuidado à PSR torna-se fundamental para a construção da resolutividade para as eCR.

A participação da comunidade local e da PSR na construção desta política pública, na perspectiva da garantia de direitos, surge da síntese final como elemento na construção do tema da resolutividade.

No contexto desta pesquisa, os trabalhadores trataram o tema dos indicadores de um modo aberto, sem distinções entre tipos de indicadores (por exemplo, de resultado ou processo), devido ao caráter exploratório do estudo. Assim, a produção da síntese do trabalho grupal é apresentada no Quadro III.

O Quadro III traz o produto do debate empreendido pelos trabalhadores sobre a temática dos indicadores. Algumas propostas na síntese foram na direção de tipos de indicadores como de autonomia, de adesão, de acessibilidade, de resolutividade, de melhoria da condição de saúde e de inserção na rede, porém não houve o apontamento concreto de como construí-los ou mesmo o que os mesmos significam exatamente para o grupo que o propôs. Na mesma linha, a produtividade das equipes também foi proposta como um indicador para o trabalho das eCR. As propostas de um modo geral escapam as indicações da literatura de que o indicador precisa ser claro para todos os envolvidos com seus usos (OPAS, 2008). 
Quadro III - Síntese sobre os indicadores.

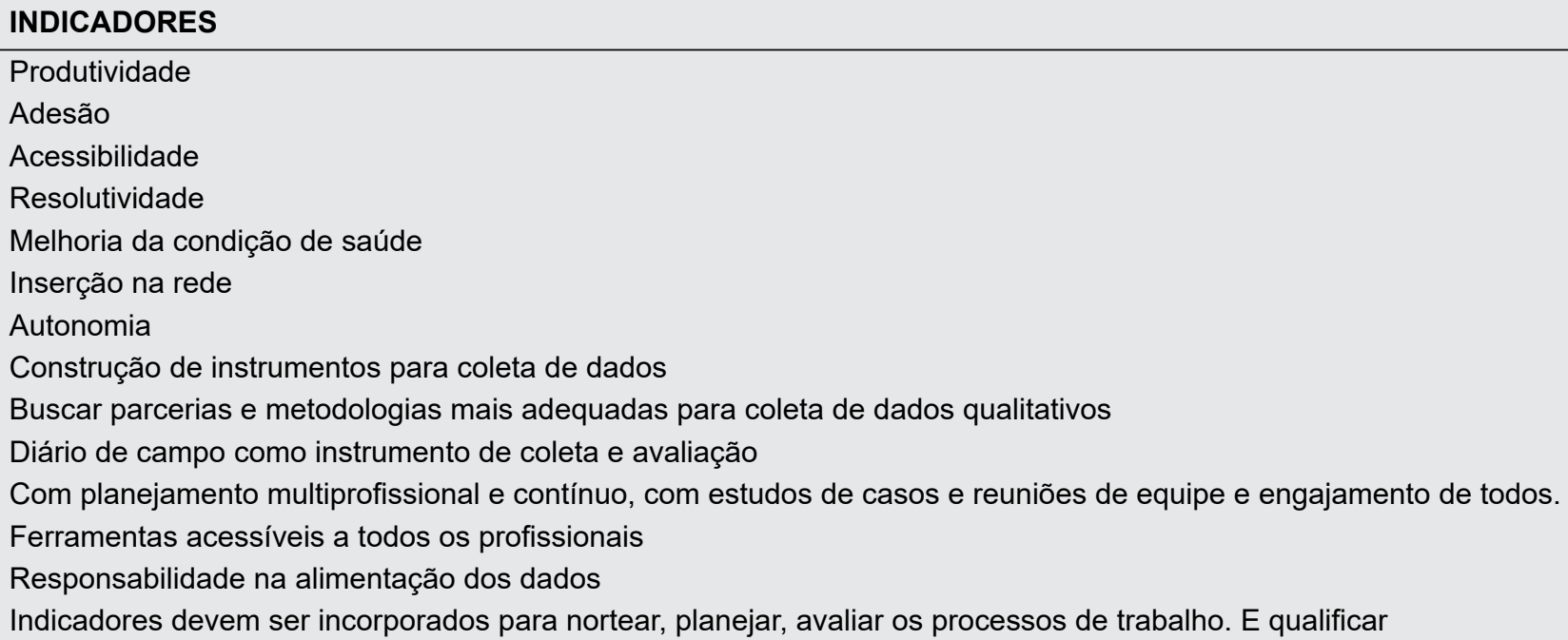

Fonte: Machado, 2017.

A síntese dos objetivos e funções dos Consultórios na Rua apresentada no Quadro IV coloca a questão da saúde como direito em vários itens, transitando pela garantia do acesso ao SUS, a inclusão social, o acesso da PSR a direitos além do campo da saúde e empoderamento da PSR. Além disso, a função de descentralizar os serviços de saúde, atender as demandas da PSR, ser resolutiva às demandas da PSR e de colocar em prática as políticas de Redução de Danos também foram apontadas pela síntese dos trabalhadores das eCR.

Quadro IV - Síntese sobre os objetivos e as funções do Consultório na Rua:

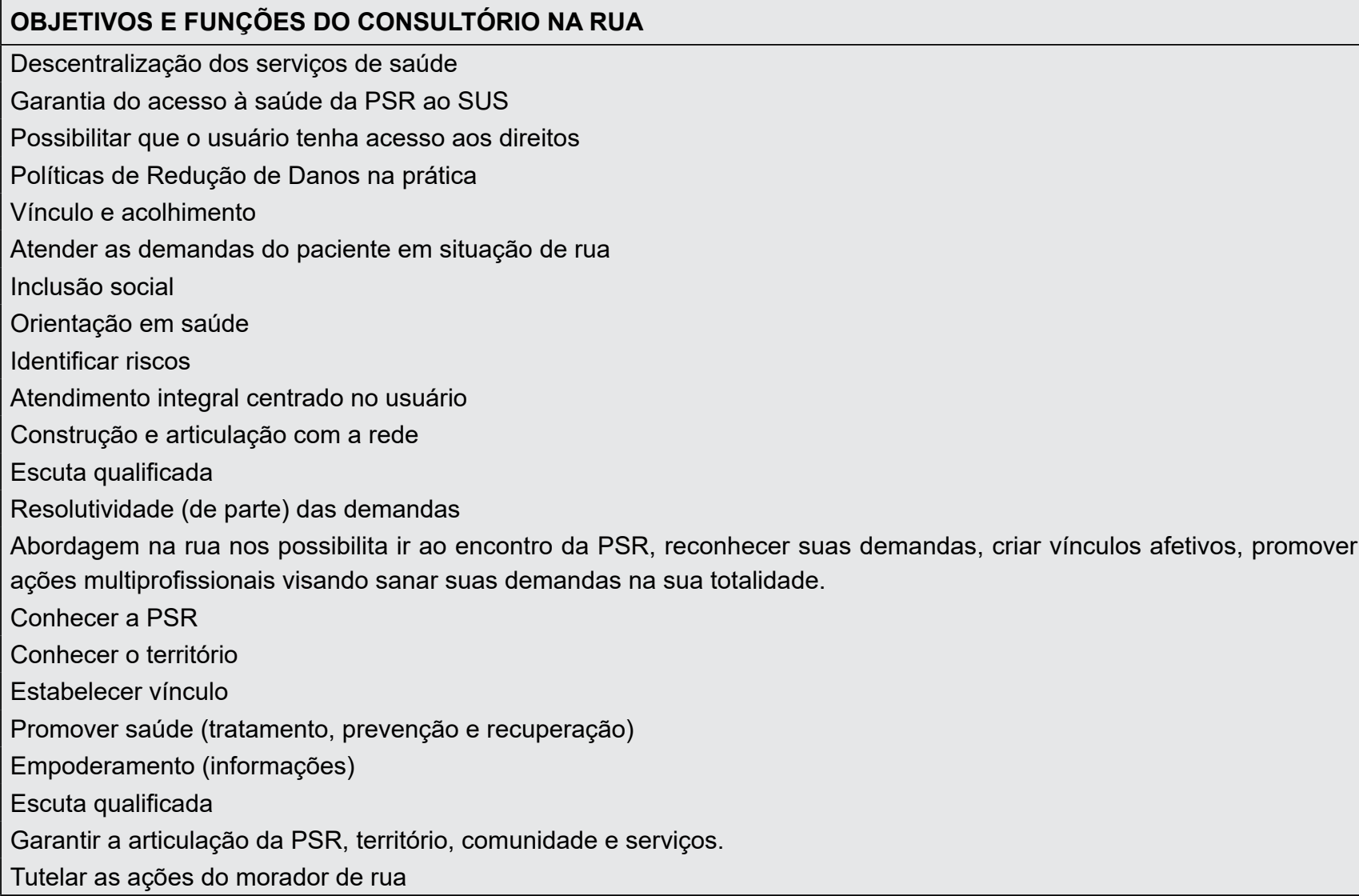

Fonte: Machado, 2017. 
No campo da clínica, a escuta qualificada, o vínculo, o acolhimento, a orientação em saúde, o atendimento integral centrado no usuário e a construção e articulação com a rede figuraram entre os objetivos e funções destas equipes.

No que tange ao processo de trabalho dos Consultórios na Rua foram apontados como objetivos: conhecer o território, estabelecer vínculo, conhecer a PSR, identificar riscos, promover saúde (tratamento, prevenção e recuperação), além de garantir a articulação da PSR, território, comunidade e serviços. Porém, um item pode causar estranhamento ou dúvida quanto a sua inserção no conjunto da síntese, o "tutelar as ações do morador de rua". Este item ganha contexto quando recorremos aos enunciados dos grupos de trabalho do World Café, onde por algumas vezes emergiram enunciados que colocavam a questão do exercício constante de equilíbrio entre tutela e autonomia que as eCR precisam praticar, devido a isso o item pode ter uma relação com um momento específico da relação com a pessoa em situação de rua, onde a eCR assume um maior protagonismo na produção do cuidado.

\section{CONCLUSÃO}

O world café foi considerado como uma técnica adequada para a coleta de dados na situação em questão por três motivos. O primeiro motivo, de ordem operacional, é que o world café coaduna com a realidade da amostra de pesquisa a ser estudada. Assim, como o universo dos sujeitos de pesquisa era de no máximo 21 trabalhadores, havia a possibilidade de adotar a referida técnica se destes, 14 confirmassem a sua participação. Nessa condição, todos os pré-requisitos foram cumpridos.

O segundo motivo, de ordem científica, indicou que o world café demonstrou ser eficaz e efetivo no processo de coleta de dados junto às equipes de saúde e evidenciou a sua relevância para a modalidade de pesquisa-ação. Isto porque permitiu que os trabalhadores interagissem, construíssem coletivamente uma visão crítica sobre suas práticas e estruturassem propostas de mudanças para o seu contexto de trabalho.

O terceiro motivo, de ordem prática e que complementa a perspectiva científica, refere-se aos efeitos do uso da técnica para os trabalhadores. Por meio da utilização do world café, foi possível observar a troca de visões e experiências, as diferentes concepções sobre os principais temas que norteiam a realidade de trabalho das equipes, as divergências em torno dos entendimentos sobre resolutividade, vulnerabilidade e indicadores e o compartilhamento das dificuldades enfrentadas pelos trabalhadores na atuação no contexto das eCRs, possibilitando através do uso desta técnica a ressignificação destes temas entre os trabalhadores.

Uma limitação que pode ser indicada na experiência aqui descrita refere-se à necessidade de demarcar de modo específico o tema a ser trabalhado para que o world café possa apresentar resultados significativos. Assim, a técnica pode não se apresentar de modo tão efetivo se o tema a ser utilizado como balizador for amplo.

Recomenda-se, assim, o amplo uso da técnica para testá-la em outras situações e verificar sua praticidade e relevância na construção de saberes coletivos. Outro elemento limitador refere-se ao número de participantes. Sugere-se que outros estudos sejam realizados com número maior de participantes para aferir se o tamanho da amostra se configura como um elemento interveniente no processo de coleta de dados.

Por fim, reforça-se o uso do world café em pesquisas pelo duplo papel que cumpre: o de investigação e coleta de dados e o de criação de um ambiente de pesquisa que estimule a reflexão sobre o saber-fazer, tornando-o, consequentemente um momento transformador do seu trabalho cotidiano.

\section{REFERÊNCIAS}

1. Bertolozzi MR, Nichiata LYI, Takahashi RF, Ciosak SI, Hino P, Val LF, Guanillo MCLTU, Pereira EG. Os conceitos de vulnerabilidade e adesão na Saúde Coletiva Rev Esc Enferm USP. 2009;43(Esp 2):1326-30.

2. Brown J, Isaacs D. The World Café: Awakening Collective Intelligence and Committed Action. In M. Torvey Collective Intelligence: Creating a Prosperous World at Peace. Earth Intelligence Network, Virginia. 2008.

3. Mandeli MC, Roland MI, Souza G, Germani AC, Gouveia N, Dias SL. Catando e reciclando saúde: Relatos do $1^{\circ}$ encontro universidade - movimento nacional de catadores de recicláveis. Cadernos Gestão Social. 2013;4(2):285-295.

4. Minayo MC, Sanches O. Quantitativo-qualitativo: Oposição ou complementaridade? Caderno de Saúde Pública. 1993;9(3):239-262.

5. Monteiro S. O marco conceitual da vulnerabilidade social. Sociedade em Debate. 2011;17(2):29-40. 
6. Rouquaryol MZ. A medida da saúde coletiva. In: epidemiologia \& saúde, $4^{\mathrm{a} e d .}$ Cap.3; p.23-71. Rio de Janeiro: Medsi, 1993.

7. Serapioni M. Métodos qualitativos e quantitativos na pesquisa social em saúde: algumas estratégias para a integração. Ciência \& Saúde Coletiva. 2000;5(1):187-192.

8. The World Café Community. Café to go! A quick reference guide for hosting world café. 2015. Disponível em http://www.theworldcafe.com/wp-content/uploads/2015/07/Cafe-To-Go-Revised.pdf

9. Turrini RNT, Lebrão ML, Cesar CLG. Resolutividade dos serviços de saúde por inquérito domiciliar: percepção do usuário. Cadernos de Saúde Pública. 2008; 24(3):663-674.

10. Merhy EE. Em busca da qualidade dos serviços de saúde: os serviços de porta aberta para a saúde e o modelo tecno-assistencial em defesa da vida (ou como aproveitar os ruídos do cotidiano dos serviços de saúde e colegiadamente reorganizar o processo de trabalho na busca da qualidade das ações de saúde). In: Cecílio LC de O, organizador. Inventando a mudança na saúde. São Paulo: Hucitec, 1994, p. 117-60.

11. Macareta IM. Traços de uma clínica de território: intervenção clínico-política na atenção básica com a rua. [Tese]. Niterói: Programa de Pós-graduação em Psicologia. Universidade Federal Fluminense; 2015.

12. Dagani VC. A resolutividade dos problemas de saúde: opinião de usuários em uma Unidade Básica de Saúde. [Dissertação]. Porto Alegre: Universidade Federal do Rio Grande do Sul; 2002.

13. D’Aguiar JMM. O Programa Saúde da Família no Brasil: a resolutividade do PSF no município de Volta Redonda (RJ). [Dissertação]. Rio de Janeiro: Mestrado em Saúde Pública. ENSP/Fiocruz; 2001.

14. Travassos C, Martins M. Uma revisão sobre os conceitos de acesso e utilização de serviços de saúde. Cadernos de Saúde Pública. 2004; 20(2):5190-8.

15. Almeida PF de, Fausto MCR, Giovanella L. Fortalecimento da atenção primária à saúde: estratégia para potencializar a coordenação dos cuidados. Rev Panam Salud Publica. 2011;29(2):84-95.

16. Chioro A, Scaff A. Saúde e cidadania: a implantação do Sistema Único de Saúde. Brasília: Ministério da Saúde; 1999.

17. Organização Pan Americana da Saúde. Rede Intragencial de Informação para a Saúde [RIPSA]. Indicadores básicos para a saúde no Brasil: conceitos e aplicações. 2 ed. Brasília: OPAS; 2008.

18. Rouquayrol MZ. Medida da saúde coletiva. In: Rouquayrol MZ, Silva MGC da. Epidemiologia e saúde. 4 ed. Rio de Janeiro: Medsi; 1993. p. 23-71.

19. Machado MPM. As práticas de cuidado produzidas pelas equipes de consultório na rua no Distrito Federal: características e desafios. [Dissertação]. Brasília: Mestrado Profissional em Políticas Públicas em Saúde, Escola Fiocruz de Governo/Fiocruz Brasília, 2017.

\section{Endereço para correspondência:}

Marcelo Pedra Martins Machado

Escola Fiocruz de Governo, Fundação Oswaldo Cruz - Brasília

Av. L3 Norte, s/n

Campus Universitário Darcy Ribeiro, Gleba A

CEP: 70.904-130 - Brasília - DF - Brasil

E-mail: marcelopedra@bol.com.br 